\title{
SAR image target detection in complex environments based on improved visual attention algorithm
}

\author{
Shuo Liu* and Zongjie Cao
}

\begin{abstract}
A novel target detection algorithm for synthetic aperture radar (SAR) images based on an improved visual attention method is proposed in this paper. With the development of SAR technology, target detection algorithms are confronted with many difficulties such as a complicated environment and scarcity of target information. Visual attention of the human visual system can make humans easily focus on key points in a complex picture, and the visual attention algorithm has been used in many fields. However, existing algorithms based on visual attention models cannot obtain satisfactory results for SAR image target detection under complex environmental conditions. After analysing the existing visual attention models, we combine the pyramid model of visual attention with singular value decomposition to simulate the human retina, which can make the visual attention model more suitable to the characteristics of SAR images. We introduce variance weighted information entropy into the model to optimize the detection results. The results obtained by the existing visual attention algorithm for target detection in SAR images yield a large number of false alarms and misses. However, the proposed algorithm can improve both the efficiency and accuracy of target detection in a complicated environment and under weak-target conditions. The experimental results validate the performance of our method.
\end{abstract}

Keywords: Visual attention; Singular value decomposition; Variance weighted information entropy (WIE); Target detection; SAR image

\section{Introduction}

The visual attention model for synthetic aperture radar (SAR) image target detection plays a positive role because the human visual system focuses on the areas of interest and rapidly decides on them [1]. Visual attention greatly improves the ability of the human visual system to deal with images under a complex environment. Therefore, a positive effect can be gained by introducing visual attention into target detection in SAR images.

With the continuous development in military technology, target detection in a SAR image becomes more difficult. The environment around targets becomes more complicated, and information on targets lessens. Traditional detection methods for SAR images such as constant false alarm rate (CFAR) have been improved to adapt different conditions, but they are also difficult to perform outstandingly. The reason is that under a

\footnotetext{
* Correspondence: Islsfox@126.com

School of Electronic Engineering, University of Electronic Science and Technology of China, Chengdu 611731, China
}

complicated environment and weak-target condition, key pixels are lacking and interference pixels are too much. Limited improvements cannot compensate for defects. Existing visual attention models generally use the Gaussian pyramid model proposed by Burt and Crouely to simulate the feature of the human retina [2]. The feature can be described by considering that the centre of the retina has a smaller receptive field; on the other hand, the receptive field at the periphery of the retina is much bigger [3]. Therefore, we can conclude that as the feature sampling density and visual resolution of a position become smaller with the increase in distance from the centre of the human retina, the peripheral information is compressed [4]. After building a Gaussian pyramid model, the visual attention system guides the attention to an area of interest according to some features of a target, such as the shape, colour and intensity [5]. Current visual attention methods are not suitable for target detection in a complicated environment or under weaktarget conditions [6].

\section{勿}


The Gaussian pyramid model of visual attention suffers from the difficulty of effectively compressing a SAR image with weak targets. But compressing an image to different rates and keeping important information is of importance for the visual attention algorithm. Therefore, this paper proposes a new visual attention algorithm for target detection. Since the singular value decomposition (SVD) method can keep the important information of a SAR image when the image is compressed [7], combining it with the Gaussian pyramid model can produce images with different compression ratios, which enable the image to retain the target information and well obscure the environment information. After combining SVD with the Gaussian pyramid model, the variance weighted information entropy (WIE) method is used to distinguish the different types of areas and filter out the regions of interest (ROIs) without targets. As a result, the purpose of efficient target detection under a complicated environment and weak targets can be achieved.

The remainder of this paper is structured as follows: Section 2 introduces in detail the classic visual attention model and the pyramid model. Section 3 presents the steps of each detection stage and focuses on the SVD method and the variance WEI. Section 4 presents the experimental results of the SAR target extraction using the proposed techniques, and Section 5 draws the conclusion.

\section{Classic visual attention model}

The algorithm proposed in this paper is an improvement of Itti. Itti is a classic visual attention model. The Itti model determines the ROI in human eyes, which includes the target to be detected as a set of significant pixels in images, and then extracts the ROI by finding a significant pixel in the image [8-10]. The model can adaptively detect the ROIs in the image. Compared with most of the traditional algorithms that need to manually specify the ROI, the Itti model enjoys great advantages in target detection and recognition in image processing. Here, we first introduce the calculation process of the Itti model, and the details can be found in the literature [11]. Then, we describe the details on building the pyramid model.

For an image, the first part of the visual attention model is the linear filter, which extracts the colour, intensity and orientation features from the image. For example, the Itti model makes the output of the Gabor filter in four different directions become the orientation feature. The formula for the Gabor filter is expressed as follows:

$$
\begin{aligned}
h\left(x, y, \theta_{\mathrm{i}}, \lambda, \alpha, \beta\right)= & \frac{1}{2 \pi \alpha \beta} \exp \left(-\pi\left(\left(\frac{x_{\theta_{i}}}{\alpha}\right)^{2}+\left(\frac{y_{\theta_{i}}}{\beta}\right)^{2}\right)\right) \\
& \exp \left(\frac{2 \pi i x_{\theta_{i}}}{\lambda}\right)
\end{aligned}
$$

where $\alpha$ and $\beta$ are the standard deviation, $\lambda$ is the wavelength and $\theta$ is the direction. The formulas of $x_{\theta_{i}}$ and $y_{\theta_{i}}$ are shown as follows:

$$
\begin{aligned}
& x_{\theta_{i}}=x \cos \left(\theta_{i}\right)+y \sin \left(\theta_{i}\right) \\
& y_{\theta_{i}}=-x \sin \left(\theta_{i}\right)+y \cos \left(\theta_{i}\right)
\end{aligned}
$$

Then, we build a Gaussian pyramid for every feature. In the second part, a centre-surround difference module is used to extract the feature map. In the third part, a plurality of different feature maps is merged to form the conspicuity map, which is a saliency map, through an effective feature consolidation strategy. In the fourth part, the focus of the attention area is located based on the saliency map. In the final part of the visual attention model, the winner-take-all competition net is used to find the most significant point from the saliency map, and the inhibition-of-return is used to ensure that the area would not be focused again [12-14].

The visual attention model adopts the multi-scale spatial attention to simulate the nonuniform sampling mechanism of the human retina [15]. Burt and Crouely proposed a pyramid structure from the summary of the nonuniform sampling mechanism [16]. Figure 1 shows the pyramid structure of an image.

Two steps are needed to establish the pyramid structure: one is smoothing and the other is downsampling. For a two-dimensional digital image $I(i, j), \delta$ represents the pyramid layers. When $\delta=0$, the formula $I_{\delta}(i, j)=I_{0}$ $(i, j)=I$ indicates the bottom of the pyramid structure.

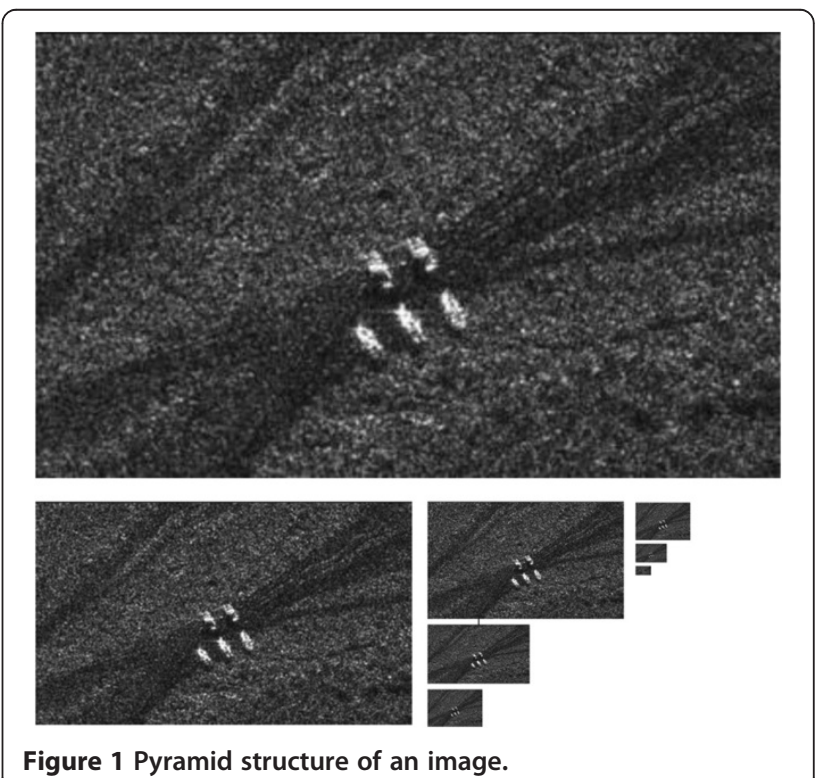


Hence, the calculation of the pyramid layers is shown as follows:

$$
I_{\sigma}(i, j)=\sum_{k=-N}^{k=N} \sum_{t=-N}^{t=N} \psi(k, t) I_{\sigma-1}(2 i+k, 2 j+t)
$$

The Itti model adopts the linear discrete Gaussian filter to perform the smoothing and downsampling in the horizontal and vertical directions of the input image, respectively, and forms eight different resolution subimages [17]. Including the original image, nine images are required to build up the Gaussian pyramid structure. The smoothing filter is [ $\left[\begin{array}{lllll}1 & 4 & 6 & 4 & 1\end{array}\right]$, and downsampling is achieved by a convolution with a [1 1$] / 2$ filter. We use the two filters to achieve our objective, which take the average value of every two pixels in the previous image as one pixel value in the next image. The two steps can be combined into a convolution with a filter $K=\left[\begin{array}{llll}1 & 4 & 6 & 4\end{array}\right.$ $1]^{*}\left[\begin{array}{ll}1 & 1\end{array}\right] / 2$ in the horizontal and vertical directions.

\section{Improved visual attention model}

In recent years, the SVD algorithm has been widely studied. This algorithm extracts the algebraic feature from an image [18]. SVD has the characteristics of energy aggregation for an image [19], which makes it a popular technology in the area of image compression. The features represent the essential characteristics of an image; therefore, the SVD algorithm has the advantage of being insensitive to noise and complexity of an image. The variance WEI is a statistical form of the characteristics, which reflects the average information of a figure. It was first used to detect infrared images [20].

\subsection{SVD integrated into visual attention}

The SVD technique has been paid close attention since its introduction and has been widely used in statistical analysis, signal processing and system theory. The SVD is an extension of the spectral analysis theory. It is essentially an orthogonal transformation. If a matrix has a linearly correlated row or column, it can be changed into a diagonal matrix by multiplying the orthogonal matrix on the left with that on the right [21]. After the change, we can obtain some singular values whose number reflects the independent row (column) vector number of the original matrix. The eigenvalues can construct each individual component of the original signal. The SVD technique has the advantage of fast processing and handing stability. The SVD in modern linear algebra and signal processing theory is defined as follows: if $A \in R^{m \times n}$ exists, then orthogonal matrices $U \in R^{m \times n}$ and $V \in R^{m \times n}$ exist. The formula is obtained as follows:

$$
A=U \Sigma V^{T} \quad\left(A=U \Sigma V^{H}\right)
$$

$$
\begin{aligned}
& \sum=\left[\begin{array}{cc}
\sum_{r} & 0 \\
0 & 0
\end{array}\right] \\
& \sum_{r}=\operatorname{diag}\left(\sigma_{1}, \sigma_{2}, \ldots, \sigma_{r}\right)
\end{aligned}
$$

The diagonal elements are arranged in a descending order:

$$
\sigma_{1} \geq \sigma_{2} \geq \cdots \geq \sigma_{n} \geq 0, r=\operatorname{rank}(A)
$$

$U$ and $V$ are $m \times m$ and $n \times n$ dimensional unitary matrices which respectively satisfy

$$
\begin{aligned}
& U U^{H}=I_{m} \\
& V V^{H}=I_{n}
\end{aligned}
$$

To make the visual attention model adjust to the target detection in a complicated-environment SAR image, we combine SVD with the pyramid model. This combination can compress the original image to a number of images with different compression rates. The information about the targets in the resulting images with different compression rates is retained and that on the environment is obscured. This result allows the centresurround module to find the saliency point more effectively and optimise the detection results under a complicated environment.

The major steps of the proposed SVD-pyramid model method are as follows:

- For an original image $A$, it is decomposed into a diagonal matrix $B$ and another two orthogonal matrices using SVD.

- The number of nonzero elements $R$ is computed, and the elements are arrayed in a descending order to form vector $P$.

- The $m$ number of biggest elements from vector $P$ is retained. The remaining elements form vector $Q$. The value of $m$ is equal to $60 \%$ of $R$.

- A new diagonal matrix $B 1$ is constructed using the elements of vector $Q$.

- A new image $A 1$ is generated using the diagonal matrix $B 1$ and the two orthogonal matrices.

- The above steps are repeated until the number of nonzero elements is less than 1 or $R \times 0.02$.

\subsection{Post-processing based on the WIE algorithm}

Entropy reflects the average information of a figure. The one-dimensional graph entropy can be expressed as

$$
H=\sum_{i=0}^{255} p i \log p i
$$

The variance WIE was first used to detect infrared images [18]. Thus, targets in infrared images alway show 


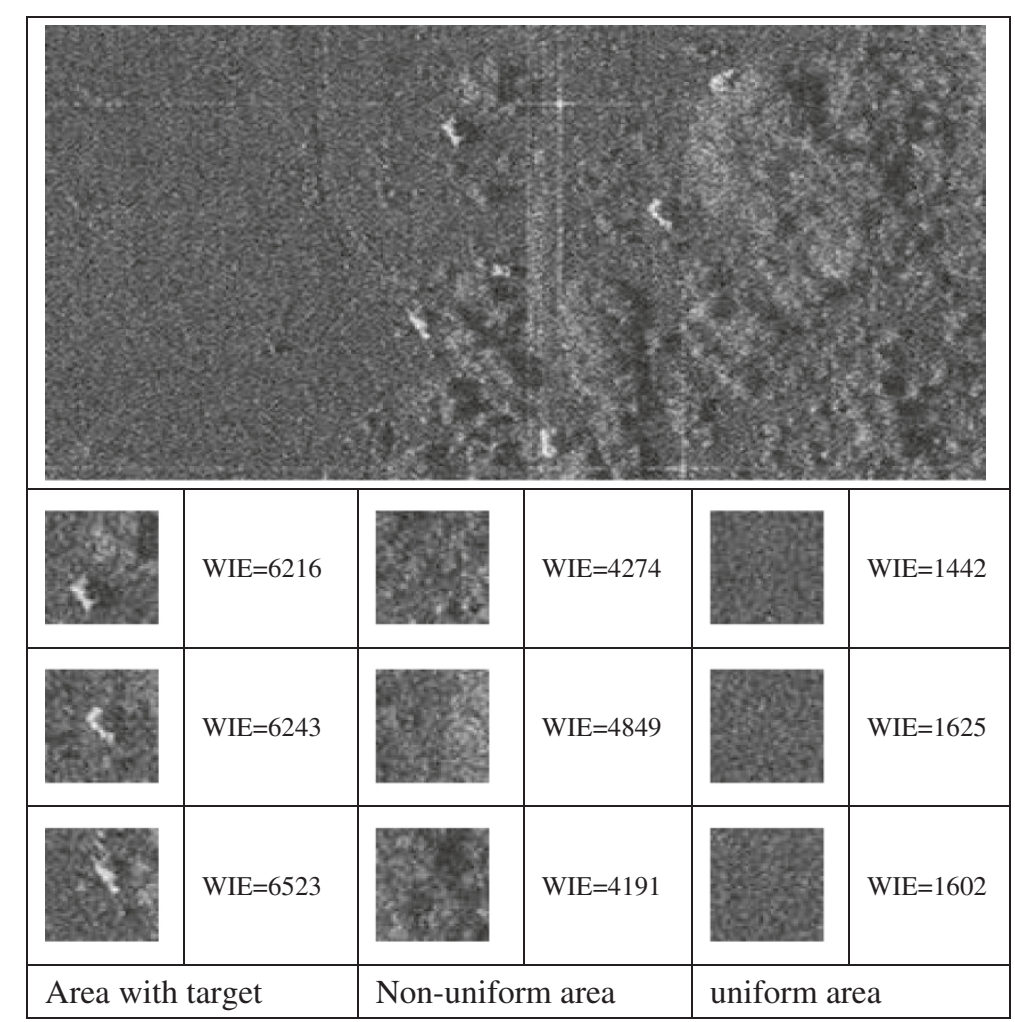

Figure 2 The WIE value with different types of areas.

different radiation and usually have a distinct grey value. The WIE algorithm is defined as follows:

$$
H(s)=-\sum_{s=0}^{255}(s-\bar{s})^{2} \text { Ps } \log (\text { Ps })
$$

where Ps is the probability of the grey levels in the infrared image and $\bar{s}$ is the mean intensity of the infrared image. In particular, when Ps $=0$, we let Ps $\log (\mathrm{Ps})=0$. Considering that the result of the improved visual attention method needs to filter out the false alarms, in this study, we import WIE to achieve this purpose. We test the value of WIE from different areas in a real SAR image. The results show that different types of regions correspond to a large difference in the WIE value, and similar types of regions correspond to a small difference in the WIE value. When the ROI areas are detected, we compute the values of WIE of these areas and array them in a descending order. Then, we calculate the differences between adjacent values and array them in an ascending order. Next, we abandon several largest values in sequence 2 and calculate the mean value in sequence 2 as a threshold value. The values in the threshold value region are corresponding to areas with a target. From this result, we can filter out the area without targets. Figure 2 shows the simulation results. The size of all

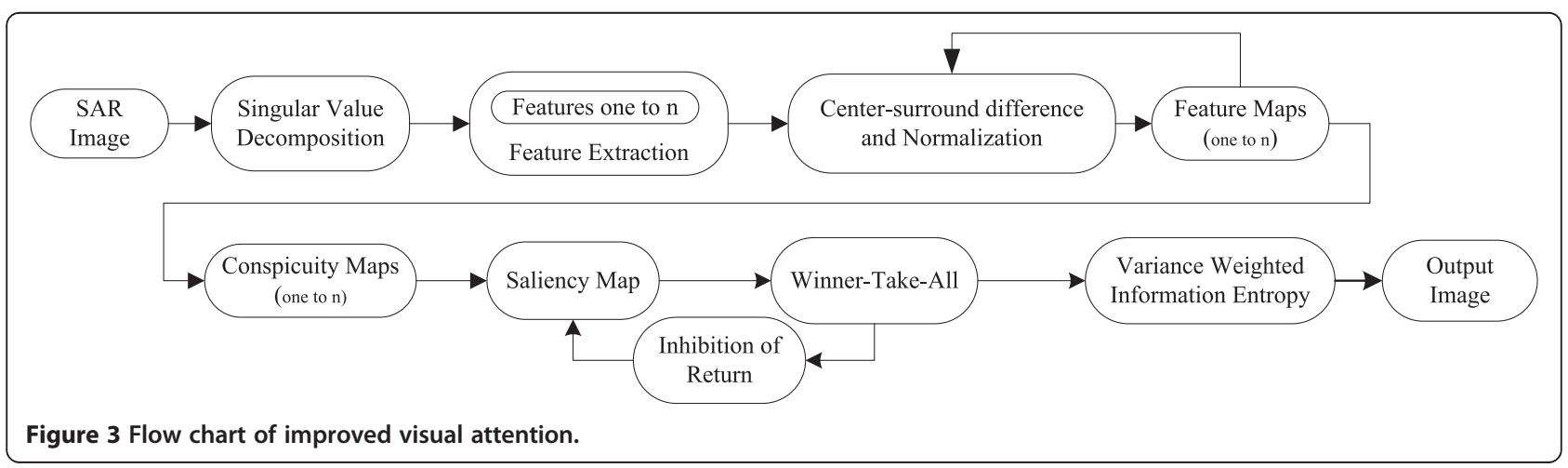




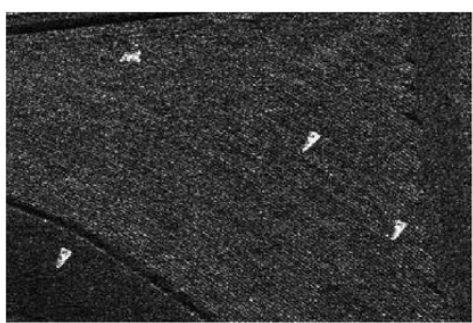

(a)

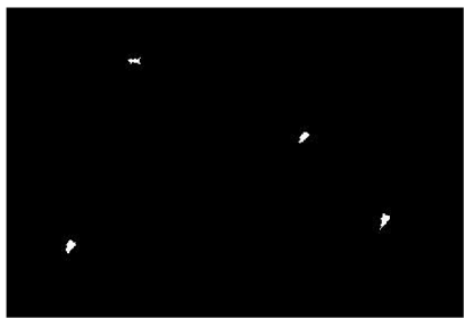

(c)

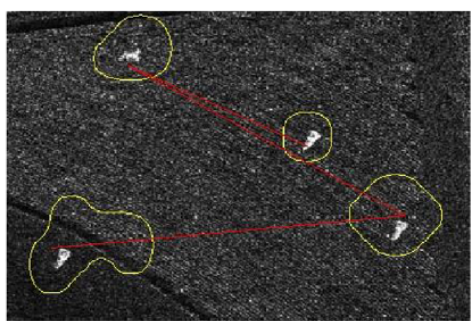

(b)

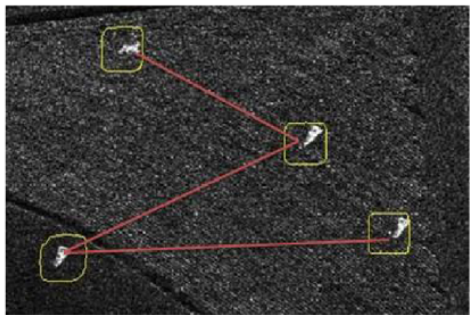

(d)

Figure 4 The test image and results by different algorithms in a simple environment. (a) The test image of a simple environment. (b) The result of the Itti model algorithm in a simple environment. (c) The result of the two-parameter CFAR method in a simple environment. (d) The result of the improved VA algorithm in a simple environment.

samples is $45 \times 45$ pixels. The sample is divided into three types, which are the area that includes the up and down, the area that contains the target and the uniform area.

The above results show that the WIE value of a real SAR image in different areas has a large difference. Based on the difference, we can determine the areas without targets. Therefore, the WIE method can be used to achieve the post-processing of the target detection.

\subsection{Processing steps}

The flow chart of the visual attention based on the SVD algorithm and WIE method mentioned earlier is shown in Figure 3.

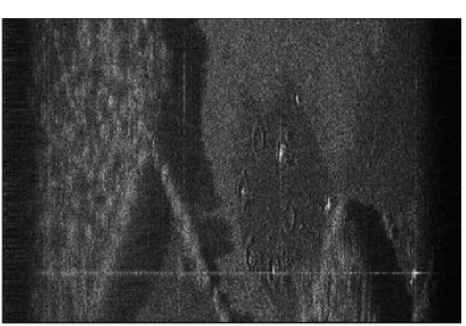

(a)

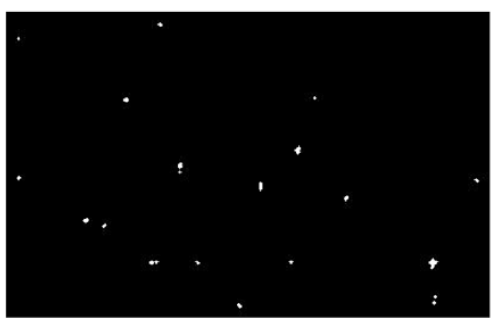

(c)

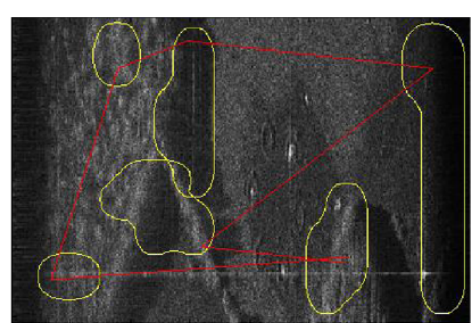

(b)

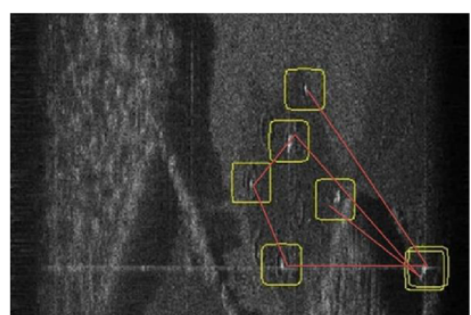

(d)

Figure 5 The results of test image 1 by different algorithms in a complicated environment. (a) The test image of a complicated environment. (b) The result of the Itti model algorithm in a complicated environment. (c) The result of the two-parameter CFAR method in a complicated environment. (d) The result of the improved VA algorithm in a complicated environment. 


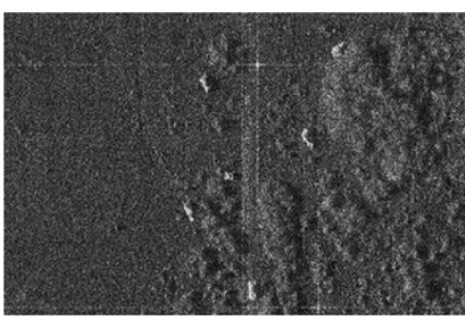

(a)

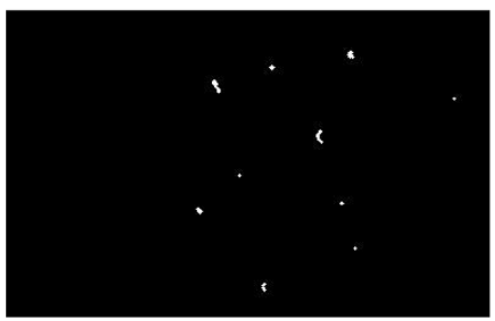

(c)

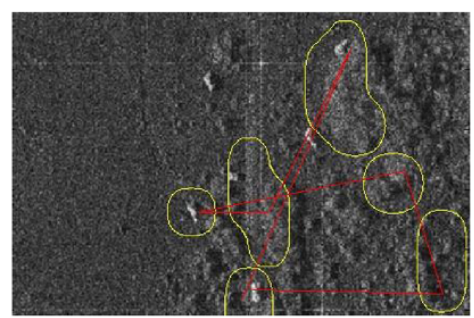

(b)

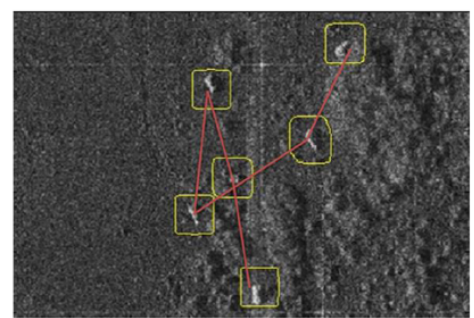

(d)

Figure $\mathbf{6}$ The results of test image $\mathbf{2}$ by different algorithms in a complicated environment. (a) The test image of a complicated environment. (b) The result of the Itti model algorithm in a complicated environment. (c) The result of the two-parameter CFAR method in a complicated environment. (d) The result of the improved VA algorithm in a complicated environment.

Because the algorithm employed in this study is based on the Itti model, the detailed steps of the algorithm will not be presented. In the feature extraction module, we use the features that include the intensity, colour, orientation and consistency. In the centre-surround difference module, the no. 2, 3 and 4 images are selected as the centre image, and the numbers of the surround images are 2, 3 and 4 .

\section{Simulation and results}

\subsection{Simulation}

To verify the feasibility of the proposed algorithm, several simulations are performed. The simulation data are divided into two types, i.e. type 1 and type 2 . Type 1 consists of 20 images, and the size of each image is $384 \times 256$. These are images in a simple environment and conspicuous target conditions. These targets are tanks in a grassland environment. Type 2 consists of images in a complicated environment and weak targets, and the size of each image is $2,406 \times 512$. The targets are tanks in grassland and jungle environment.

The simulations are divided into three parts. The first part is the simulation using the Itti model. The second part is the simulation using the proposed algorithm. The last part is the simulation using the two-parameter CFAR. This detection method is a pixel-level target detection method. The reason we choose CFAR for comparison is that the algorithm is not only the most in-depth and most practical but also an extensive method of a class at present. The simulation of each part satisfies two premises, i.e. simple environment and conspicuous target condition and complicated environment and weak-target condition. Figure 4a shows the test image for the first type of data, which are under the simple environment and conspicuous target conditions. Figure $4 \mathrm{~b}, \mathrm{c}, \mathrm{d}$ shows the results obtained by the three algorithms. In the figures, red lines and yellow lines show the shifting route and size of FOA, respectively.

To verify the performance of the improved algorithm under a complicated environment, we perform another simulation using the type 2 data. Figures $5 \mathrm{a}$ and $6 \mathrm{a}$ show the test images of the type 2 data. They are obtained at different levels of complexity condition. Figures $5 b, c, d$ and $6 \mathrm{~b}, \mathrm{c}, \mathrm{d}$ show the results obtained by the three algorithms.

Table 1 Whole performance comparisons among the three algorithms in a simple environment

\begin{tabular}{lcccr}
\hline & $\begin{array}{c}\text { Number of } \\
\text { all targets }\end{array}$ & $\begin{array}{c}\text { Number of } \\
\text { detected targets }\end{array}$ & $\begin{array}{c}\text { False } \\
\text { alarms }\end{array}$ & $\begin{array}{c}\text { Number of } \\
\text { undetected targets }\end{array}$ \\
\hline Classic algorithm & 4 & 4 & 0 & 0 \\
CFAR & 4 & 4 & 0 & 0 \\
The proposed algorithm & 4 & 4 & 0 & 0 \\
\hline
\end{tabular}


Table 2 Whole performance comparisons among the three algorithms in a complicated environment

\begin{tabular}{lccccc}
\hline & $\begin{array}{c}\text { Number of } \\
\text { all targets }\end{array}$ & $\begin{array}{c}\text { Number of } \\
\text { detected targets }\end{array}$ & $\begin{array}{c}\text { False } \\
\text { alarms }\end{array}$ & $\begin{array}{c}\text { Number of } \\
\text { undetected targets }\end{array}$ & $\begin{array}{c}\text { Size of FOA (number } \\
\text { of pixels) }\end{array}$ \\
\hline Classic algorithm & 13 & 12 & 6 & 7 & 2,000 to 9,000 (large) \\
CFAR & 13 & 27 & 14 & 0 & - \\
The proposed algorithm & 13 & 13 & 0 & 0 & 1,000 to 1,500 (small) \\
\hline
\end{tabular}

\subsection{Results}

In the two data types for the simulation, we obtain the following results by comparing the three algorithms. The results are divided into two parts. First, for the data in the simple environment and weak target case, the results are listed in Table 1. The three methods yield four focus of attention (FOA) areas, and the four areas detect the targets well. All three methods do not yield false alarms and undetected targets. We compare the size of the FOA for two kinds of visual attention algorithm. The size of the FOA is measured by the number of pixels in the FOA. Through the simulation, the number of pixels in the FOA in the classic algorithm is 2,000 to 7,000 , and it is 1,000 to 1,500 in the proposed algorithm. Therefore, the size of the FOA in the improved method is smaller.

From the type 2 data obtained by the simulation, we obtain the results listed in Table 2. Six targets are present in the SAR image. From the classic algorithm, in the six FOA areas obtained by the Itti model, only three areas cover the targets. The others cover shadow and empty areas. The two-parameter CFAR method detected ten targets. Although the six targets are detected, four false alarms occur. On the other hand, all six target areas obtained by the proposed algorithm exactly cover the targets. In the classic algorithm, the number of pixels in the FOA is 2,000 to 9,000, and in the proposed algorithm, it is 1,000 to 1,500 . Thus, the FOA size in the improved method is also smaller.

By comparing the detected rates, we determine that the performance of the classic algorithm is significantly lower than that of the other two algorithms and the proposed algorithm performs best. From the comparison of the false alarm rates, the false alarm rate and misses of the classic algorithm are high. Although the CFAR method successfully detects all the targets, the false alarm rate is higher than that of the proposed method. The detected rate is defined as the ratio of the number of correct detected target to real target. The false alarm rate is defined as the ratio of the number of false detected target to real target. By comparing the three methods, the classic method produces too many false alarms which affect the detection accuracy. Itti model methods miss a lot of targets, but the proposed method can make up for the lack. In summary, the proposed algorithm can obtain not only a high detection rate but also a low false alarm rate. The improved visual attention algorithm can adapt to the conditions of a complicated environment and weak target.

After comparing the performance of the three methods, we study the effect of image complexity on the detection performance of the three methods. We use the GLCM features to describe the spatial distribution and whole complexity of an image. The features include the angular second moment, entropy, inverse different moment and contrast. Figure 7 shows the result.

Figure 7 shows that the detection rate of the classic method rapidly decreases. Because the complexity of the image increases, the background becomes complicated, and the classic method detects more complicated regions than real targets. The detection rate of the CFAR method decreases slowly. It is based on a large number

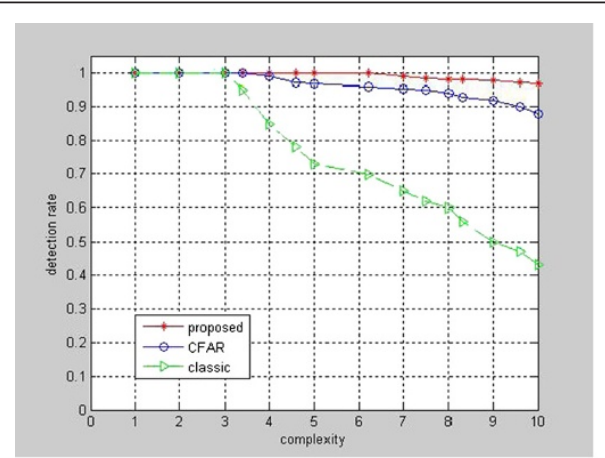

(a)

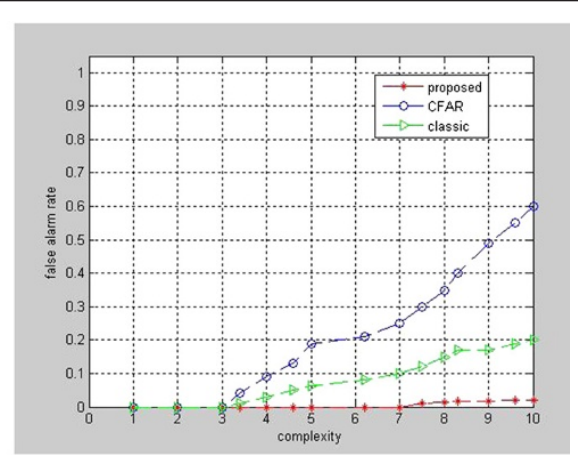

(b)

Figure 7 Detection results of the three methods in different complexities. (a) Detection rate. (b) False alarm rate. 
of false alarms. With the increase in complexity, the detection efficiency rapidly decreases. The proposed method yields better results in both detection and false alarm rates.

\section{Conclusions}

In this study, we have developed an improved visual attention algorithm adapted to SAR image target detection under complicated environment and weak-target conditions. The method of combining SVD with the pyramid model and using WIE to filter out the false alarms has been introduced in detail. To validate the performance of the method, some simulations were performed. The results show the feasibility of the improved visual attention algorithm to SAR image target detection under complicated environment conditions.

\section{Competing interests}

The authors declare that they have no competing interests.

\section{Acknowledgements}

This work was supported in part by the National Natural Science Foundation of China under Projects 60802065, 61271287 and 61371048

Received: 2 January 2014 Accepted: 26 March 2014

Published: 5 April 2014

\section{References}

1. DB Walther, C Koch, Attention in hierarchical models of object recognition. Prog. Brain Res. 165, 57-78 (2007)

2. L Itti, C Koch, Computational modeling of visual attention. Nat. Neurosci. 2, 194-203 (2001)

3. D Walther, Interactions of visual attention and object recognition: computational modeling, algorithms, and psychophysics. PhD thesis (California Institute of Technology, Pasadena, CA, 2006)

4. JK Tsotsos, Y Liu, J Martinez-Trujillo, M Pomplun, E Simine, K Zhou, Attending to visual motion, computer vision and image understanding. Spec. Issue Attention Perform. Comput. Vision 100, 3-40 (2005)

5. V Navalpakkam, L Itti, An integrated model of top-down and bottom-up attention for optimal object detection, in Proc. IEEE Conference on Computer Vision and Pattern Recognition (CVPR) (New York, June 2006), pp. 2049-2056

6. J Feng, Z Cao, Y Pi, Multiphase SAR image segmentation with G0 statistical model based active contours. IEEE Trans. GRS 51(7), 4190-4199 (2013)

7. IA Rybak, VI Gusakova, AV Golovan, LN Podladchikova, NA Shevtsova, A model of attention-guided visual perception and recognition. Vis. Res. 38, 2387-2400 (1998)

8. S Shah, MD Levine, Visual information processing in primate cone pathways-part. I. A model, systems, man, and cybernetics. IEEE Trans. 2, 259-274 (1996)

9. Z Li, L Itti, Saliency and gist features for target detection in satellite images. IEEE Trans. Image Process. 20(7), 2017-2029 (2011)

10. R Desimone, J Duncan, Neural mechanism of selective visual attention. Annu. Rev. Neurosci. 18, 193-194 (1995)

11. S Lee, K Kim, JY Kim, M Kim, HJ Yoo, Familiarity based unified visual attention model for fast and robust object recognition. Pattern Recogn. 43(3), 1116-1128 (2010)

12. AM Treisman, G Gelade, A feature-integration theory of attention. Cogn. Psychol. $12,97-136(1980)$

13. K Cater, A Chalmers, G Ward, Detail to attention: exploiting visual tasks for selective rendering, in Proc. 14th Eurographics Workshop Rendering (Eurographics Association, Aire-la-Ville, 2003), pp. 270-280

14. L Itti, C Koch, E Niebur, A model of saliency-based visual attention for rapid scene analysis, pattern analysis and machine intelligence. IEEE Trans. 20, 1254-1259 (1998)

15. I Rigas, G Economou, S Fotopoulos, Low-level visual saliency with application on aerial imagery. Geosci. Remote Sens. Lett. IEEE 10, 1389-1393 (2013)
16. A Borji, L Itti, Exploiting local and global patch rarities for saliency detection, in Proc. IEEE Conference on Computer Vision and Pattern Recognition (Providence, 2012), pp. 478-485

17. D Walther, U Rutishauser, C Koch, P Perona, Selective visual attention enables learning and recognition of multiple objects in cluttered scenes. Comput Vision Image Understanding 100(1-2), 41-63 (2005)

18. H Andrews, C Patterson, Singular value decomposition (SVD) image coding. IEEE Trans. Commun. 24(4), 425-432 (1976)

19. B Arnold, An investigation into using singular value decomposition as a method of image compression (Department of Mathematics and Statistics, University of Canterbury, 2000)

20. L Yang, Y Zhou, J Yang, Variance WIE based infrared images processing. Electron. Lett. 42(15), 857-859 (2006)

21. R Ruotolo, C Surace, Using SVD to detect damage in structures with different operational conditions. J. Sound Vib. 226(3), 425-439 (1999)

doi:10.1186/1687-1499-2014-54

Cite this article as: Liu and Cao: SAR image target detection in complex environments based on improved visual attention algorithm. EURASIP Journal on Wireless Communications and Networking 2014 2014:54.

\section{Submit your manuscript to a SpringerOpen ${ }^{\odot}$ journal and benefit from:}

- Convenient online submission

- Rigorous peer review

- Immediate publication on acceptance

- Open access: articles freely available online

- High visibility within the field

- Retaining the copyright to your article

Submit your next manuscript at $\gg$ springeropen.com 